\title{
Chitosan hydrogel improves mesenchymal stem cell transplant survival and cardiac function following myocardial infarction in rats
}

\author{
BIN XU, YANG LI, BO DENG, XIAOJING LIU, LIN WANG and QING-LEI ZHU \\ Department of Cardiology, Chinese PLA Hospital, Beijing 100853, P.R. China
}

Received September 11, 2015; Accepted November 4, 2016

DOI: $10.3892 / \mathrm{etm} .2017 .4026$

\begin{abstract}
Myocardial infarction (MI) remains the leading cause of cardiovascular-associated mortality and morbidity. Improving the retention rate, survival and cardiomyocyte differentiation of mesenchymal stem cells (MSCs) is important in improving the treatment of patients with MI. In the present study, temperature-responsive chitosan hydrogel, an injectable scaffold, was used to deliver MSCs directly into the infarcted myocardium of rats following MI. Histopathology and immunohistochemical staining were used to evaluate cardiac cell survival and regeneration, and cardiac function was assessed using an echocardiograph. It was demonstrated that chitosan hydrogel increased graft size and cell retention in the ischemic heart, promoted MSCs to differentiate into cardiomyocytes and increased the effects of MSCs on neovasculature formation. Furthermore, chitosan hydrogel enhanced the effect of MSCs on the improvement of cardiac function and hemodynamics in the infarcted area of rats following MI. These findings suggest that chitosan hydrogel is an appropriate material to deliver MSCs into infarcted myocardium.
\end{abstract}

\section{Introduction}

There have been numerous advances in medical and surgical procedures; however, coronary heart disease remains a key cause of cardiovascular-associated mortality and morbidity (1). Following acute myocardial infarction (MI), the heart undergoes remodeling and left ventricular (LV) function decreases due to a limited capacity of self-renewal in the heart (2). The progression of adverse ventricular remodeling, compromises the cardiac function of patients with MI and 10-40\% develop fatal end-stage heart failure (3-5). Current medical therapies exhibit limited efficacy for heart failure and heart

Correspondence to: Professor Qing-Lei Zhu, Department of Cardiology, Chinese PLA Hospital, 28 Fuxing Road, Beijing 100853, P.R. China

E-mail: qlzhu@yahoo.com

Key words: chitosan hydrogel, mesenchymal stem cells, myocardial infarction, cardiac function, cardiac hemodynamics transplantation is problematic due to a shortage of donors (6). Therefore, the development of novel therapies, particularly stem cell based therapy, to treat heart failure is required.

Mesenchymal stem cells (MSCs) are pluripotent, adult stem cells that are found within the microenvironment of the bone marrow (7). The use of MSCs is advantageous as they are easy to isolate, possess the potential to differentiate into multiple lineages and have extensive ex vivo expansion capacity. Therefore, MSCs have been widely used in experimental animal (8) and clinical studies (9), for cardiac repair and regeneration following MI. However, the effectiveness of MSCs in the heart has not yet been fully elucidated; MSC therapy in post-MI animal models has demonstrated only modest or transient functional improvements $(10,11)$. This is may be due to lower retention rates, low survival rates of transplanted MSCs and inefficient cardiomyocyte differentiation of MSCs in vivo $(12,13)$.

Chitosan is a natural cationic copolymer and is an amino polysaccharide copolymer comprised of 1, 4-D-glucosamines and N-acetyl glucosamines (14). As a scaffold protein, chitosan hydrogel has been widely used in the agriculture, food and pharmaceutical industries, and has specifically been used in the engineering of tissues, including skin, nerves, bone, cartilage, blood vessels and liver (15). Lu et al (14) determined that chitosan hydrogel was also an injectable scaffold that improved the retention of embryonic stem cells in post-MI rats. However, whether chitosan hydrogel is beneficial to MSC retention, thus enhancing their role in improving cardiac function, remains unknown.

The present study used temperature-responsive chitosan hydrogel, which facilitates the adherence of MSCs, as an injectable scaffold to deliver MSCs. The retention and survival of implanted MSCs within the infarcted myocardium were subsequently assessed. Combination therapy with chitosan hydrogel and MSCs was also assessed, with regards to improving cardiac cell survival, cardiac function and its mechanism, using histopathology, immunohistochemical staining and echocardiography.

\section{Materials and methods}

Isolation, culture and identification of rat bone marrow MSCs. Bone marrow MSC cultures were prepared according to previously described methods (16). Four 2-month old 
female pathogen-free Sprague-Dawley rats weighing 240-260 g were purchased from the Experimental Animal Center, Chinese PLA General Hospital (Beijing, China). Rats were housed at a temperature of $20-23^{\circ} \mathrm{C}$, humidity of 40-70\%, had ad libitum access to food and water and were maintained on a $12 \mathrm{~h}$ light-dark cycle. Rats were euthanized with a pentobarbital (Sigma-Aldrich; Merck Millipore, Darmstadt, Germany) overdose ( $200 \mathrm{mg} / \mathrm{kg}$ ), approved by the Animal Care and Use Committee of Chinese PLA General Hospital (Beijing, China). The femur and tibia were excised under sterile conditions, with special attention given to the removal of all connective tissue attached to bones. Bone marrow plugs were extracted by flushing the bone marrow cavity with complete culture medium made up of low sugar Dulbecco's Modified Eagle's medium (Gibco; Thermo Fisher Scientific, Inc., Waltham, MA, USA) supplemented with $10 \%$ fetal bovine serum (Gibco; Thermo Fisher Scientific, Inc.) and $1 \%$ penicillin-streptomycin (Sigma-Aldrich; Merck Millipore). Titrating the solution produced a widely dispersed marrow plug suspension. Following centrifugation at $37^{\circ} \mathrm{C}$ of a homogenous cell suspension ( $220 \mathrm{x} \mathrm{g}$ for $8 \mathrm{~min}$ ), cells were resuspended in complete culture medium and incubated at $37^{\circ} \mathrm{C}$ in a humidified atmosphere with $5 \% \mathrm{CO}_{2}$ for three days prior to the first medium change. The medium was changed 5 times overall. The mesenchymal population was harvested on the basis of their ability to adhere to the culture plate (17). When the cells reached $90 \%$ confluence, they were sub-cultured with $0.25 \%$ trypsin-EDTA (Sigma-Aldrich; Merck Millipore) into $225 \mathrm{~cm}^{2}$ flasks at a ratio of 1:3. First-passage MSCs were used in all experiments. Prior to injection, cultured MSCs were stained with $5 \mu \mathrm{M}$ DAPI (Sigma-Aldrich; Merck Millipore) for $30 \mathrm{~min}$ at $37^{\circ} \mathrm{C}$, washed with phosphate-buffered saline (PBS) and collected for use.

Cultured MSCs were analyzed by fluorescence-activated cell sorting using an Epics XL cytometer (Beckman Coulter, Inc., Brea, CA, USA). Cells were incubated at $37^{\circ} \mathrm{C}$ for $30 \mathrm{~min}$ with phycoerythrin-conjugated mouse monoclonal antibodies against rat cluster of differentiation (CD) 29 (1:200, catalogue number 12-0291, eBioscience, Inc., San Diego, CA, USA), CD44 (1:200, catalogue number 12-0444, eBioscience, Inc.), CD11b (1:200, catalogue number 12-0110, eBioscience, Inc,.), and CD34 (1:250, catalogue numberH6-NB600-1071, Novus Biologicals, LLC., Littleton, CO, USA). The same-species, same-isotype irrelevant antibodies served as negative controls including rat immunoglobulin ( Ig) G (1:1,000, catalogue number 12-4301, eBioscience, Inc.) and IgG2aк (both 1:1,000, catalogue numbers 12-4321 and H6-NBP1-43780, eBioscience Inc. and Novus Biologicals, LLC.).

Production of the MI animal model. To establish a model of MI, 48 male pathogen-free Sprague-Dawley rats (weights, 225-250 g) were obtained from Experimental Animal Center of Chinese PLA General Hospital (Beijing, China). All rats were specific-pathogen-free and kept in a controlled environment at $20-23^{\circ} \mathrm{C}$ and $40-70 \%$ humidity in a ventilated rack with a 12 h light-dark cycle. Food and water were given ad libitum. The rat model of MI was produced as previously described (18). Male Sprague-Dawley rats were anesthetized with ketamine $(90 \mathrm{mg} / \mathrm{kg})$ and xylazine $(10 \mathrm{mg} / \mathrm{kg}$; both Sigma-Aldrich; Merck Millipore). Under sterile conditions, pericardial sac removal was performed through a median sternotomy. While the landmarks ( $2 \mathrm{~mm}$ under the edge of the left atrial appendage) on the left atrium base and the interventricular groove were kept in view, a single stitch of 7-0 Ticron suture was placed through the myocardium at a depth slightly greater than the perceived level of the left coronary artery, taking care not to enter the ventricular chamber. The chest was subsequently closed and the animals were allowed to recover for one week. All protocols approved by the Animal Care and Use Committee of Chinese PLA General Hospital (Beijing, China).

Preparation of temperature-responsive chitosan hydrogel. Temperature-responsive chitosan hydrogel was prepared as previously reported (19). A total of $200 \mathrm{mg}$ chitosan (catalogue number, 448877; degree of deacetylation, 84\%; molecular weight, 292,000; Sigma-Aldrich; Merck Millipore) was dissolved in $10 \mathrm{ml}$ distilled water to obtain a $2 \%$ chitosan solution. Following this, $1.15 \mathrm{~g} \beta$-glycerophosphate disodium ( $\beta$-GP; Sigma-Aldrich; Merck Millipore) was dissolved in $10 \mathrm{ml}$ distilled water to produce an $11.5 \% \beta$-GP solution, which was filter sterilized. Subsequently, temperature-responsive chitosan hydrogel was produced by mixing $8 \mathrm{ml}$ of $2 \%$ chitosan, $2 \mathrm{ml}$ of $11.5 \% \beta$-GP solution and $2.5 \mathrm{ml}$ of $2.5 \%$ (w/v in Dulbecco's modified Eagle medium) filter-sterilized hydroxyethyl cellulose (Sigma-Aldrich; Merck Millipore) and incubating the solution at $37^{\circ} \mathrm{C}$ for $20 \mathrm{~min}$.

Injection surgeries. The 48 rats were randomized to either control or treatment groups $(n=38)$ and treated with one of four regimens one week after MI: $50 \mu 1$ PBS ( $\mathrm{n}=10$, control), $50 \mu \mathrm{l}$ chitosan hydrogel $(\mathrm{n}=10), 1 \times 10^{7} \mathrm{MSCs}$ in $50 \mu \mathrm{l}$ of $0.5 \%$ PBS $(\mathrm{n}=14)$ or $1 \times 10^{7} \mathrm{MSCs}$ in $50 \mu \mathrm{l}$ chitosan hydrogel $(n=14)$. The treatment was injected into the infarcted myocardium. Under sterile conditions, the rats were anesthetized with ketamine $(90 \mathrm{mg} / \mathrm{kg})$ and xylazine $(10 \mathrm{mg} / \mathrm{kg})$ and the abdomens were opened from the xiphoid process to a left subaxillar level along the lower rib. The LV apex was exposed via a subdiaphragmatic incision, leaving the chest wall and sternum intact. Injections were administered using a 30-gauge needle, into multiple sites of the LV infarcted wall and central area. The ischemic area was identified by the characteristic appearance of a darker region of the LV with limited contractility.

Immunofluorescent and immunohistochemical staining. A total of 24 rats were euthanized with a pentobarbital (Sigma-Aldrich; Merck Millipore) overdose $(200 \mathrm{mg} / \mathrm{kg}$ ) at either $24 \mathrm{~h}$ or 5 weeks after cell transplantation. At 24 h, 8 rats treated with MSCs in PBS $(n=4)$ or chitosan hydrogel $(n=4)$ were selected randomly and at 5 weeks, 16 rats in 4 groups (4 rats from each group) were selected randomly for euthanasia. The hearts were rapidly excised and frozen in Tissue Tek O.C.T. freezing medium (Sakura Finetek USA, Inc., Torrance, CA, USA).

A total of 5 sections (10- $\mu$ m thick), equally distributed through the infarct area, were obtained from each heart as representative samples. To measure cell retention within the myocardium, the area covered by the DAPI-labeled MSCs was traced using a fluorescent microscope (Olympus BX51, 
Olympus Corp., Tokyo, Japan) and measured using RS ImagePro version 4.5 (Media Cybernetics, Inc., Rockville, MD, USA). The percentage of DAPI-labeling in the infarct area was determined as cell retention.

To detect the differentiation of rat MSCs in infarcted hearts of mice sacrificed 5 weeks post-transplantation, immunofluorescence staining was performed with monoclonal rat anti-cardiac actin (1:100, catalogue number ZM-0003, Beijing Zhongshan Jinqiao Biotechnology Co., Ltd., Beijing, China). To monitor the density of microvessels, the sections underwent immunohistochemistry with factor VIII-related antigen antibody (1:100, catalogue number ZM0022, Beijing Zhongshan Jinqiao Biotechnology Co., Ltd.) The following criteria were used to assess the assay: i) Positive for vessel endothelium labeling; ii) having a visible lumen; iii) within the infarct scar; and iv) possessing a diameter of $10-100 \mu \mathrm{m}$.

Echocardiograph studies. Echocardiograph studies were performed four weeks after cell transplantation by an investigator blinded to treatment allocation.

Echocardiograms were performed on the 24 remaining rats anesthetized with ketamine $(90 \mathrm{mg} / \mathrm{kg}$ ) with an echocardiographic system equipped with a 7.5-MHz transducer (HP Sonos 5500; HP, Inc., Palo Alto, CA, USA) (20). LV dimensions were measured as previously described (21). Fractional shortening (FS) was calculated using the following formula: (LVDd-LVDs)/LVDdx100, where LVDd is LV diastolic dimension and LVDs is LV systolic dimension.

Hemodynamic studies. Hemodynamic studies were performed five weeks after cell transplantation. A 1.5F micromanometer-tipped catheter (Millar, Inc., Houston, TX, USA) was inserted into the right carotid artery to measure the mean arterial pressure (22). The catheter was subsequently inserted into the LV to measure LV pressure. Hemodynamic variables were measured with a pressure transducer (TSD104A) connected to an MP150 BIOPAC ${ }^{\circledR}$ system (Biopac systems, Inc., Goleta, CA, USA).

Statistical analysis. Numerical values are expressed as mean \pm standard error of the mean, unless otherwise indicated. Comparisons of parameters between two groups were performed with unpaired Student $t$-test. Comparisons of parameters among groups were determined by one-way analysis of variance, followed by the Scheffe multiple-comparison test. $\mathrm{P}<0.05$ was considered to indicate a statistically significant difference.

\section{Results}

Characterization of cultured MSCs. The majority of cultured MSCs expressed CD29 and CD44, and were negative for CD34 and CD11b (Fig. 1).

Cell retention and graft size. Cells injected with chitosan hydrogel covered $18.5 \pm 2.0 \%$ of the infarct area (Fig. 2A), whereas only $3.9 \pm 0.4 \%$ coverage was observed in the PBS group (Fig. 2B). This indicates that there was a significant increase in the number of MSCs in the chitosan hydrogen group $24 \mathrm{~h}$ after injection, compared with the PBS group $(n=4$;
$\mathrm{P}<0.01$; Fig. 2C). These data indicated that chitosan hydrogel increased cell retention and graft size in the infarcted heart. MSCs injected with PBS were frequently scattered in the infarct areas, whereas those injected with chitosan hydrogel were primarily in aggregates (Fig. 2A and B).

Differentiation of MSCs in infarcted hearts. Following treatment with chitosan hydrogel, MSCs or a combination of chitosan hydrogel and MSCs for four weeks, the expression of actin was increased in the infarct area in all three groups (Fig. 3). However, actin expression in the group receiving combination treatment was markedly more increased as compared with the other groups (Fig. 3D), indicating that chitosan hydrogel promoted MSCs to differentiate into myocytes.

Effects of the combination of chitosan hydrogel and MSCs on cardiac function and hemodynamics. Results from echocardiographic studies demonstrated LV dysfunction in the infarcted group, as indicated by a decrease in ejection fraction (EF) and FS. Following treatment with chitosan hydrogel, MSCs or a combination for four weeks, significant increases $(\mathrm{P}<0.05)$ in EF and FS were observed, when compared with the infarcted group. In addition, the increase was much higher in the combined chitosan hydrogel and MSCs group compared with the chitosan hydrogel, or the MSCs only group (Fig. 4A and $\mathrm{B} ; \mathrm{n}=6, \mathrm{P}<0.05$ ). Left ventricular systolic pressure and LV maximum dp/dt were significantly lower in the infarcted group than in the sham group $(\mathrm{P}<0.01)$. However, these variables significantly improved following treatment with chitosan hydrogel, MSCs or a combination for 4 weeks (Fig. 4C and D; $\mathrm{n}=6, \mathrm{P}<0.05$ ). Furthermore, LV end-diastolic pressure indicated a significant elevation in the infarcted group, compared with the sham group $(\mathrm{P}<0.01)$. However, this elevation was significantly attenuated in the treatment groups, particularly in the group treated with a combination of MSCs and chitosan hydrogel (Fig. 4E; $n=6, P<0.05)$. The results suggested that chitosan hydrogel enhanced the effect of MSCs, improving cardiac function and hemodynamics in the infarcted area.

Effects of combining chitosan hydrogel and MSCs on neovasculature formation. In the present study, detection of factor VIII-related factors was used to assess neovasculature formation. The results demonstrated that, compared with the infracted group (Fig. 5A), treatment with chitosan hydrogel alone (Fig. 5B), MSCs alone (Fig. 5C) and chitosan hydrogel combined with MSCs (Fig. 5D), all increased microvessel density in scar areas $(\mathrm{n}=4 ; \mathrm{P}<0.01)$. Among these groups, the microvessel density of the chitosan hydrogel combined with MSCs was the highest, being significantly increased compared with both the infarcted and MSCs alone groups (Fig. 5E; $n=4 ; \mathrm{P}<0.05$ ). The chitosan hydrogen alone and MSCs alone groups were significantly increased compared with the infarcted group $(\mathrm{P}<0.05)$.

\section{Discussion}

In the current study, it was demonstrated that chitosan hydrogel increases MSC retention and graft size in the ischemic heart, promotes MSC differentiation into myocytes, increases the 
A

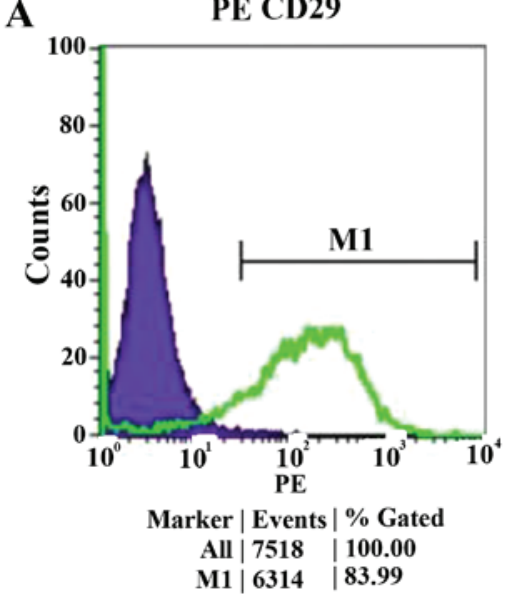

C

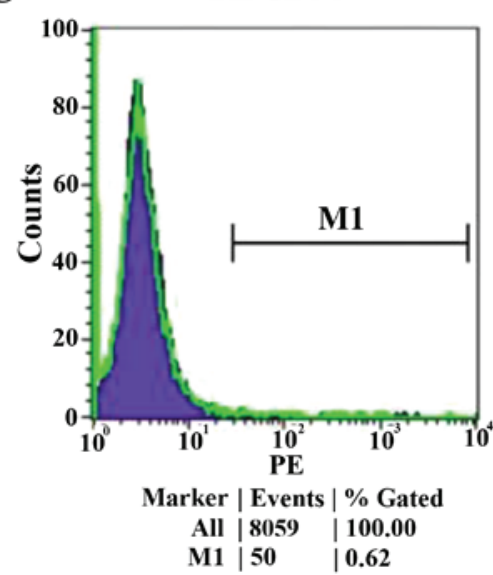

B

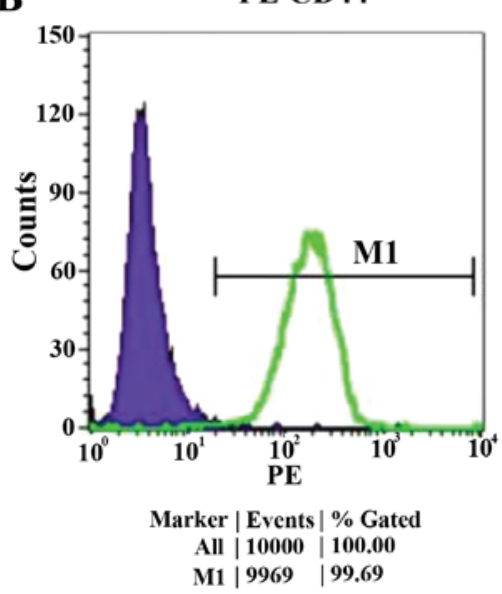

D

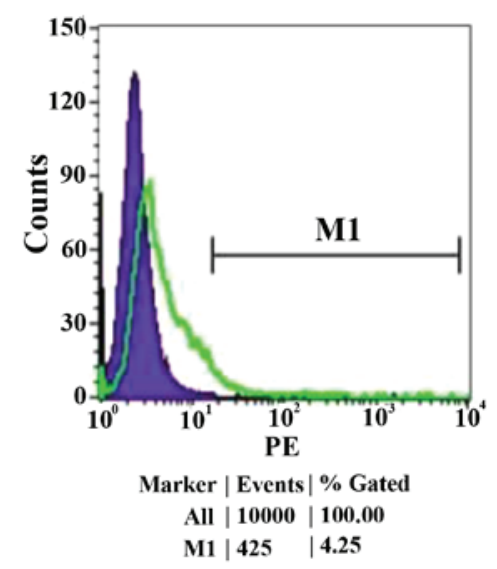

Figure 1. Flow-cytometric analysis of the adherent MSC population expanded to four and five passages. The majority of the MSCs expressed (A) CD29 and (B) CD44; however, they were negative for (C) CD31 and (D) CD11b. CD, cluster of differentiation; MSCs, mesenchymal stem cells. PE, Phycoerythrin; M1, negative control peak.
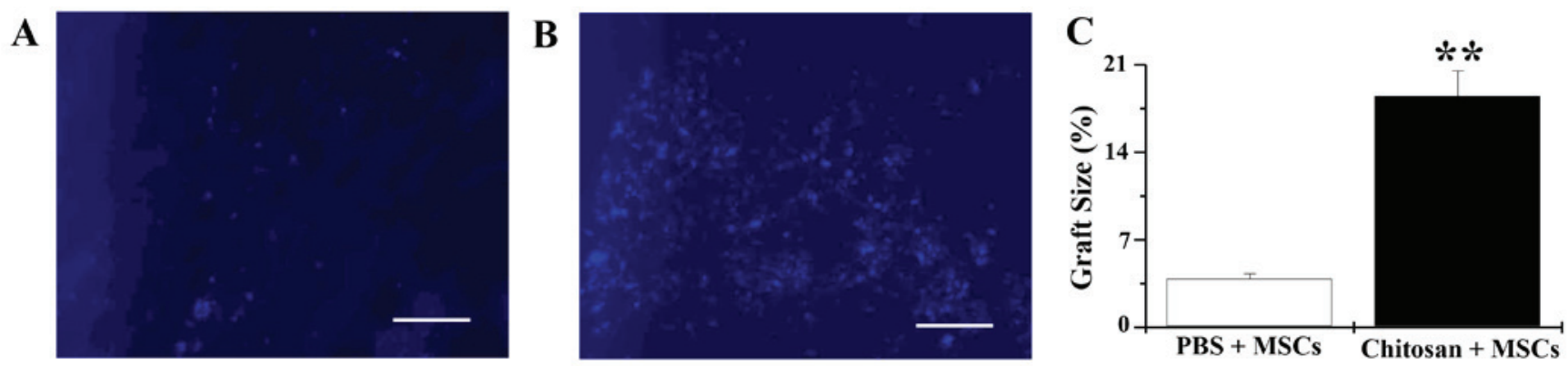

Figure 2. Chitosan enhances rat MSC retention rate $24 \mathrm{~h}$ following transplantation. (A) PBS + MSCs group: DAPI-labeled MSCs are scattered in the infarct area. (B) Chitosan + MSCs group: DAPI-labeled MSCs are predominantly accumulated in the aggregate. (C) Percentage size of MSCs within the infarct area. $\mathrm{n}=4$. ${ }^{* *} \mathrm{P}<0.01$ vs. PBS+MSCs group. Bars represent $50 \mu \mathrm{m}$. MSC, mesenchymal stem cells; PBS, phosphate buffered saline.

effects of MSCs on neovasculature formation and enhances the effect of MSCs on the improvement of cardiac function and hemodynamics following $\mathrm{MI}$ in rats.

Previous studies have demonstrated that transplantation of MSCs improves cardiac function in experimental models of ischemic heart disease $(23,24)$. The present study indicated that transplantation of MSCs alone induced differentiation into myocytes and improved cardiac function in post-MI rats, which was consistent with previous findings (25). However, there are a number of problems associated with the transplantation of MSCs that influence the outcome of stem cell transplantation for MI treatment, including a high death rate of transplanted MSCs in the infarcted area and low efficiency of differentiation into cardiomyocytes (26). It has been demonstrated that $>90 \%$ of translated cells undergo apoptosis or necrosis $24 \mathrm{~h}$ following transplantation (27). Therefore, it is critical to develop a strategy 

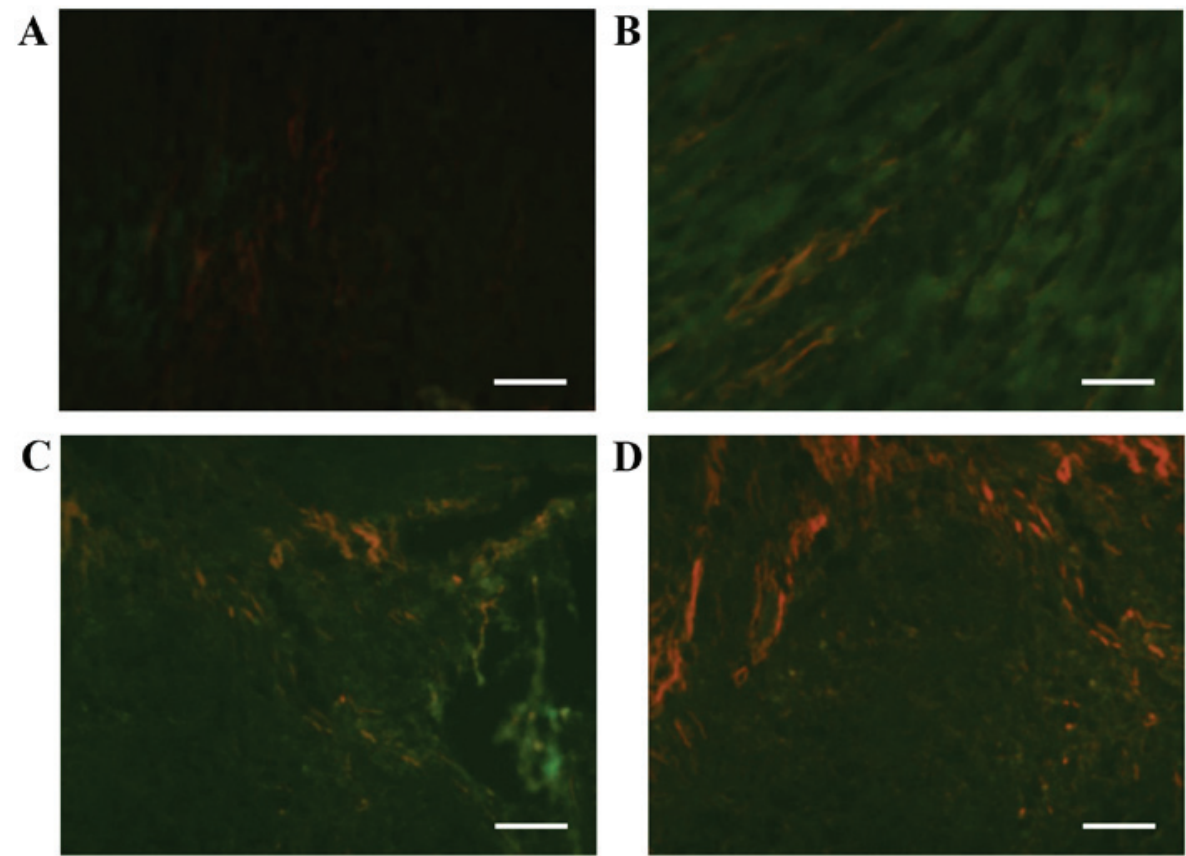

Figure 3. Differentiation of rat MSCs in infarcted hearts. Cardiac actin immunofluorescence staining of DAPI-labeled MSCs four weeks following transplantation revealed DAPI-positive and actin-positive cells in the infarct area. (A) Infarcted group, (B) chitosan alone, (C) MSCs alone and (D) combined chitosan and MSC treatment. Red represents actin; bars represent $100 \mu \mathrm{m}$. MSC, mesenchymal stem cells.

A

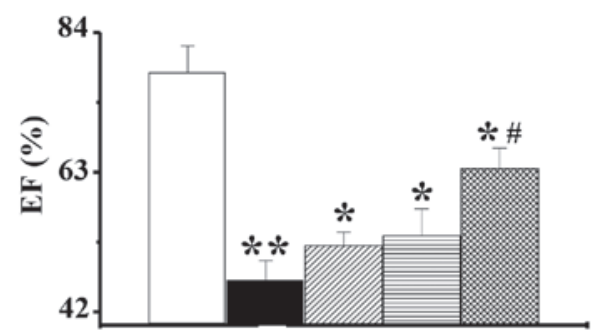

C

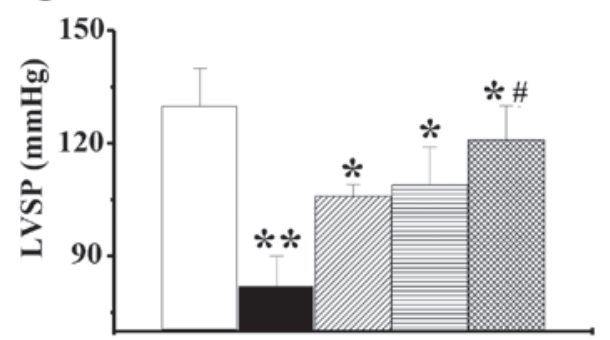

$\mathbf{E}$

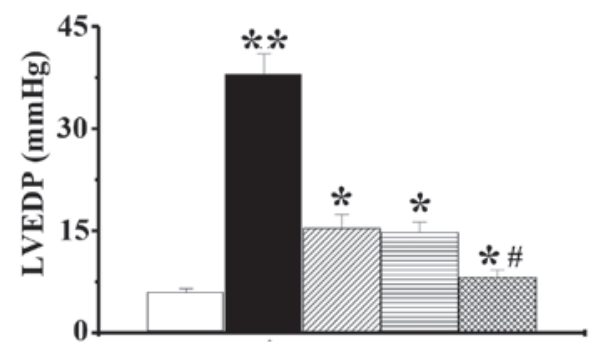

B

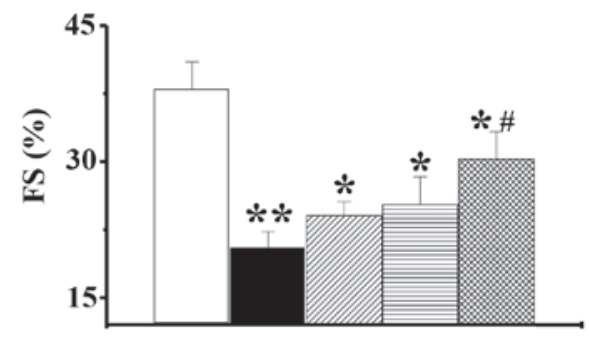

D

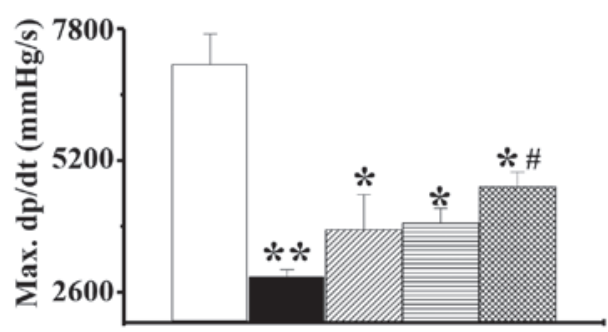

Figure 4. Heart function was improved in the rats that were treated with chitosan, rat MSCs and combined chitosan and MSCs. The combined chitosan and MSCs group demonstrated a significantly higher heart function than the groups treated with chitosan and MSC alone. Analysis of (A) ejection fraction, (B) fractional shortening, (C) left ventricular peak systolic pressure, (D) LV maximum dp/dt, and (E) LV end-diastolic pressure. $n=6$. ${ }^{* *} \mathrm{P}<0.01$ vs. sham group; ${ }^{*} \mathrm{P}<0.05$ vs. infarcted group; ${ }^{*} \mathrm{P}<0.05$ vs. MSCs alone group. MSC, mesenchymal stem cells; LV, left ventricle; EF, ejection fraction; FS, fractional shortening; LVSP, left ventricular peak systolic pressure; LVSDP, left ventricular end-diastolic pressure. 

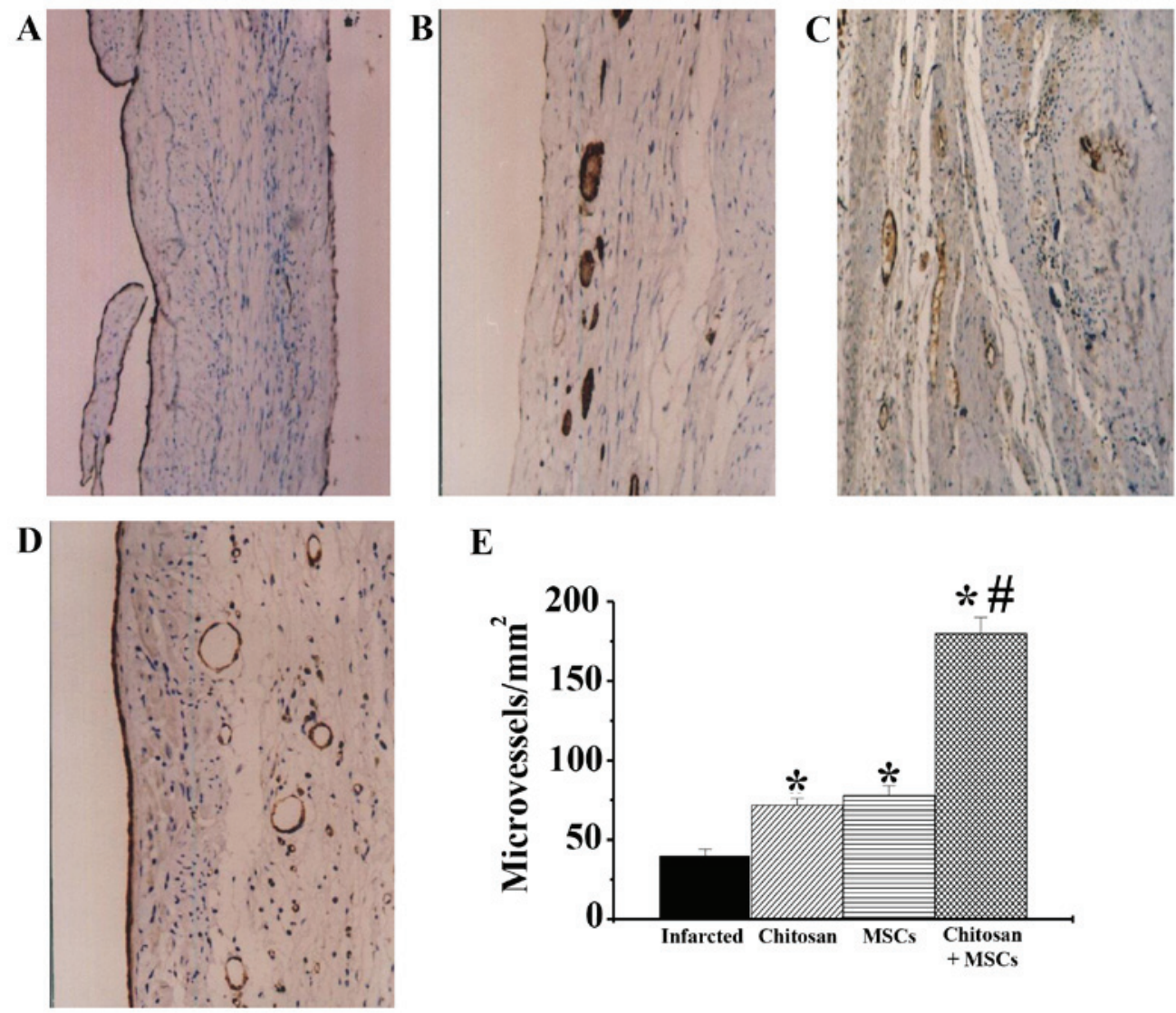

Figure 5. Effects of combined chitosan hydrogel and MSC treatment on neovasculature formation. Neovasular formation for (A) infarcted, (B) chitosan treatment, (C) MSC treatment and (D) combined treatment with chitosan and MSCs. (E) Statistical analysis. The results demonstrated that the neovasculature density was improved in the group receiving combined treatment, as compared with those receiving $\mathrm{MSCs}$ alone. $\mathrm{n}=4$. ${ }^{*} \mathrm{P}<0.05 \mathrm{vs}$. infarcted group; ${ }^{\sharp} \mathrm{P}<0.05$ vs. MSCs alone group. MSC, mesenchymal stem cells.

to improve the survival and differentiation of transplanted cells in the infarction site in order to improve the efficiency of stem cell therapy.

As it is a biocompatible and biodegradable cationic polymer, chitosan hydrogel is widely used in the agriculture, food and pharmaceutical industries $(28,29)$. The results of the present study indicate that chitosan hydrogel not only increased MSC retention and graft size in an ischemic heart, but also promoted MSCs to differentiate into myocytes, thus enhancing the effect of MSCs on the improvement of cardiac function and hemodynamics in the infarct area. Furthermore, the results indicated that chitosan hydrogel may be used as a tissue-engineering scaffold to deliver MSCs in the future.

It is well known that the microenvironment has a crucial role in the viability and function of transplanted cells. Zhang et al (26) reported that the survival of cells injected into vascularized myocardium was improved compared with cells injected into ischemic myocardium. It was also reported that accelerated angiogenesis is beneficial in the formation of normal granulation tissue during the typical course of healing (30). In the current study, chitosan hydrogel increased vessel density, enhancing the living condition of transplanted cells, thus improving cardiac function. This demonstrates that the injection of chitosan hydrogel alone or together with MSCs increased the microvessel density significantly within the infarct area, suggesting that chitosan hydrogel may be beneficial in enhancing the effects of MSCs on neovasculature formation. Ishishara et al (31) suggested that chitosan hydrogel induces vascularization in the healing process of wounds. In addition, chitosan hydrogel may be used to modulate the proliferation of vascular cells in vitro and in vivo (32). The underlying mechanism by which hydrogel increases neovascularization may be associated with the modulation of proliferation and migration of vascular cells. This is potentially an important mechanism required to improve the cardiac function of rats following MI; however, other mechanisms require future investigation for clarification.

In conclusion, the current study indicated that chitosan hydrogel increases MSC retention, promotes MSC differentiation into myocytes and increases the effects of MSCs on neovasculature formation. Thus, chitosan hydrogen enhances the improvement of cardiac function and hemodynamics following myocardial infarction and may be a potential material used for the delivery of MSCs to the infarcted myocardium.

\section{Acknowledgements}

The present study was supported by grants from the National Natural Science Foundation of China (grant nos. 81070200 and 81200157), Beijing Nova Program (grant no. 2005A064) 
and the Key Project of National Natural Science Foundation of China (grant no. 81030002).

\section{References}

1. Cohn JN: The management of chronic heart failure. N Engl J Med 335: 490-498, 1996.

2. Pfeffer MA and Braunwald E: Ventricular remodeling after myocardial infarction: Experimental. Observations and clinical implications. Circulation 81: 1161-1172, 1990.

3. Frangogiannis NG, Smith CW and Entman ML: The inflammatory response in myocardial infarction. Cardiovasc Res 53: $31-47,2002$.

4. Gordon O, Gilon D, He Z, May D, Lazarus A, Oppenheim A and Keshet E: Vascular endothelial growth factor-induced neovascularization rescues cardiac function but not adverse remodeling at advanced ischemic heart disease. Arterioscler Thromb Vasc Biol 32: 1642-1651, 2012.

5. Velagaleti RS, Pencina MJ, Murabito JM, Wang TJ, Parikh NI, D'Agostino RB, Levy D, Kannel WB and Vasan RS: Long-term trends in the incidence of heart failure after myocardial infarction. Circulation 118: 2057-2062, 2008.

6. Nakou ES and Vardas PE: New therapeutic options in heart failure. What's on the horizon? An overview. Int J Cardiol 170 95-106, 2013

7. Williams AR and Hare JM: Mesenchymal stem cells: Biology, pathophysiology, translational findings, and therapeutic implications for cardiac disease. Circ Res 109: 923-940, 2011.

8. Silva GV, Litovsky S, Assad JA, Sousa AL, Martin BJ, Vela D, Coulter SC, Lin J, Ober J, Vaughn WK, et al: Mesenchyma stem cells differentiate into an endothelial phenotype, enhance vascular density, and improve heart function in a canine chronic ischemia model. Circulation 111: 150-156, 2005.

9. Hare JM, Traverse JH, Henry TD, Dib N, Strumpf RK, Schulman SP, Gerstenblith G, DeMaria AN, Denktas AE, Gammon RS, et al: A randomized, double blind, placebocontrolled, dose-escalation study of intravenous adult human mesenchymal stem cells (prochymal) after acute myocardial infarction. J Am Coll Cardiol 54: 2277-2286, 2009.

10. Cho J, Zhai P, Maejima Y and Sadoshima J: Myocardial injection with GSK-3 $\beta$-overexpressing bone marrow-derived mesenchymal stem cells attenuates cardiac dysfunction after myocardial infarction. Circ Res 108: 478-489, 2011.

11. den Haan MC, van Zuylen VL, Pluijmert NJ, Schutte CI, Fibbe WE, Schalij MJ, Roelofs H and Atsma DE: Discrepant results of experimental human mesenchymal stromal cell therapy after myocardial infarction: Are animal models robust enough? PLoS One 11: e0152938, 2016.

12. Bartunek J, Behfar A, Vanderheyden M, Wijns W and Terzic A: Mesenchymal stem cells and cardiac repair: Principles and practice. J Cardiovasc Transl Res 1: 115-119, 2008

13. Hill JM, Dick AJ, Raman VK, Thompson RB, Yu ZX, Hinds KA Pessanha BS, Guttman MA, Varney TR, Martin BJ, et al: Serial cardiac magnetic resonance imaging of injected mesenchymal stem cells. Circulation 108: 1009-1014, 2003.

14. Lu WN, Lü SH, Wang HB, Li DX, Duan CM, Liu ZQ, Hao T, He WJ, Xu B, Fu Q, et al: Functional improvement of infarcted heart by co-injection of embryonic stem cells with temperature-responsive chitosan hydrogel. Tissue Eng Part A 15 $1437-1447,2009$

15. Ruvinov E, Sapir Y and Cohen S (eds): Cardiac Tissue Engineering: Principles, Materials and Applications. 1st edition. Synthesis Lectures on Tissue Engineering: pp1-200, 2012.

16. Barbash IM, Chouraqui P, Baron J, Feinberg MS, Etzion S, Tessone A, Miller L, Guetta E, Zipori D, Kedes LH, et al: Systemic delivery of bone marrow-derived mesenchymal stem cells to the infarcted myocardium: Feasibility, cell migration, and body distribution. Circulation 108: 863-868, 2003.
17. Tomita S, Li RK, Weisel RD, Mickle DA, Kim EJ, Sakai T and Jia ZQ: Autologous transplantation of bone marrow cells improves damaged heart function. Circulation 100: (19 Suppl) II247-II256, 1999.

18. Sievers RE, Schmiedl U, Wolfe CL, Moseley ME, Parmley WW, Brasch RC and Lipton MJ: A model of acute regional myocardial ischemia and reperfusion in the rat. Magn Reson Med 10: 172-181, 1989.

19. Chenite A, Chaput C, Wang D, Combes C, Buschmann MD, Hoemann CD, Leroux JC, Atkinson BL, Binette F and Selmani A: Novel injectable neutral solutions of chitosan form biodegradable gels in situ. Biomaterials 21: 2155-2161, 2000.

20. Nagaya N, Uematsu M, Kojima M, Ikeda Y, Yoshihara F, Shimizu W, Hosoda H, Hirota Y, Ishida H, Mori $\mathrm{H}$ and Kangawa K: Chronic administration of ghrelin improves left ventricular dysfunction and attenuates development of cardiac cachexia in rats with heart failure. Circulation 104: 1430-1435, 2001.

21. Nagaya N, Kangawa K, Itoh T, Iwase T, Murakami S, Miyahara Y, Fujii T, Uematsu M, Ohgushi H, Yamagishi M, et al: Transplantation of mesenchymal stem cells improves cardiac function in a rat model of dilated cardiomyopathy. Circulation 112: 1128-1135, 2005.

22. Okumura H, Nagaya N, Itoh T, Okano I, Hino J, Mori K, Tsukamoto $\mathrm{Y}$, Ishibashi-Ueda $\mathrm{H}$, Miwa $\mathrm{S}$, Tambara $\mathrm{K}$, et al: Adrenomedullin infusion attenuates myocardial ischemia/reperfusion injury through the phosphatidylinositol 3-kinase/Akt-dependent pathway. Circulation 109: 242-248, 2004.

23. Shake JG, Gruber PJ, Baumgartner WA, Senechal G, Meyers J, Redmond JM, Pittenger MF and Martin BJ: Mesenchymal stem cell implantation in a swine myocardial infarct model: Engraftment and functional effects. Ann Thorac Surg 73: 1919-1926, 2002

24. Min JY, Sullivan MF, Yang Y, Zhang JP, Converso KL, Morgan JP and Xiao YF: Significant improvement of heart function by cotransplantation of human mesenchymal stem cells and fetal cardiomyocytes in postinfarcted pigs. Ann Thorac Surg 74: 1568-1575, 2002

25. Wen Z, Zheng S, Zhou C, Wang J and Wang T: Repair mechanisms of bone marrow mesenchymal stem cells in myocardial infarction. J Cell Mol Med 15: 1032-1043, 2011.

26. Zhang M, Methot D, Poppa V, Fujio Y, Walsh K and Murry CE: Cardiomyocyte grafting for cardiac repair: Graft cell death and anti-death strategies. J Mol Cell Cardiol 33: 907-921, 2001.

27. Vono R, Spinetti G, Gubernator M and Madeddu P: What's new in regenerative medicine: Split up of the mesenchymal stem cell family promises new hope forcardiovascular repair. J Cardiovasc Transl Res 5: 689-699, 2012.

28. Dang JM, Sun DD, Shin-Ya Y, Sieber AN, Kostuik JP and Leong KW: Temperature-responsive hydroxybutyl chitosan for the culture of mesenchymal stem cells and intervertebral disk cells. Biomaterials 27: 406-418, 2006.

29. Olmez SS, Korkusuz P, Bilgili H and Senel S: Chitosan and alginate scaffolds for bone tissue regeneration. Pharmazie 62: 423-431, 2007

30. Ueno H, Mori T and Fujinaga T: Topical formulations and wound healing applications of chitosan. Adv Drug Deliv Rev 52: $105-115,2001$.

31. Ishihara M, Obara K, Ishizuka T, Fujita M, Sato M, Masuoka K, Saito Y, Yura H, Matsui T, Hattori H, et al: Controlled release of fibroblast growth factors and heparin from photocrosslinked chitosan hydrogels and subsequent effect on in vivo vascularization. J Biomed Mater Res A 64: 551-559, 2003.

32. Chupa JM, Foster AM, Sumner SR, Madihally SV and Matthew HW: Vascular cell responses to polysaccharide materials: in vitro and in vivo evaluations. Biomaterials 21: 2315-2322, 2000. 